\title{
Estrategia "Hands On" Y Su Incidencia En El Desarrollo De La Comprensión Lectora Del Idioma Inglés
}

\author{
Luis Even Pazmiño Pavon \\ Paul Rolando Armas Pesantez
}

Magister en Lingüística Aplicada al Aprendizaje del Idioma Inglés. Docente de la Escuela Superior Politécnica de Chimborazo, Ecuador

Centro de Idiomas

Edgar Eduardo Heredia Arboleda

Magister En Enseñanza De Ingles Como Idioma Extranjero

Docente de la Escuela Superior Politécnica de Chimborazo, Ecuador

Centro de Idiomas

\section{Doi:10.19044/esj.2018.v14n23p85 URL:http://dx.doi.org/10.19044/esj.2018.v14n23p85}

\begin{abstract}
This paper focuses on applying a strategy through a didactic guide called "Hands On" with tales, games, and vocabulary to develop the reading comprehension of English Language students of sixth level class "E" of the Language Center at Universidad Nacional de Chimborazo (UNACH), Ecuador. The research type was transversal, quasi-experimental, descriptive, and correlational. Due to observation and a checklist, it was possible to evaluate it before and after applying the strategy by means of the application of activities planned in the English subject syllabus. For this, it was necessary to take into account a sample of 30 students, identify the causes of the lack of motivation on them in order to develop the English language reading comprehension, and analyze the intellectual capacities of the group and the English Language teaching-learning methods. The data collected were tabulated, analyzed, and interpreted to be finally put under a statistical test in order to check the hypothesis. Therefore, it was concluded that the application of tales, games, and vocabulary contained in the guide influences the development of the English Language reading comprehension in a positive way.
\end{abstract}

Keywords: Strategy, Hands On, Incidence, Reading Comprehension Development, English 


\section{Resumen}

El objetivo de la presente investigación, fue aplicar una estrategia a través de una guía didáctica denominada "Hands On" con cuentos, juegos y vocabulario para el desarrollo de la comprensión lectora del idioma inglés en los estudiantes de sexto nivel "E" del Centro de Idiomas de la Universidad Nacional de Chimborazo (UNACH). La investigación fue transversal, cuasi experimental, descriptiva de tipo correlacional. Mediante la observación y una lista de cotejo se evaluó el antes y después de aplicar la estrategia, mediante la aplicación actividades programadas en el sílabo de la asignatura de inglés. Para ello se consideró una muestra de 30 estudiantes, identificando las causas que desmotivan al estudiante para desarrollar su comprensión lectora del idioma inglés, analizando las capacidades intelectuales del grupo y analizando los métodos de enseñanza - aprendizaje del idioma inglés. Los datos recolectados fueron tabulados, analizados e interpretados $\mathrm{y}$ finalmente sometidos a una prueba estadística a fin de comprobar las hipótesis, las mismas que concluyeron que la aplicación de cuentos, juegos y vocabulario contenidos en la guía didáctica, incide positivamente en el desarrollo de la comprensión lectora del idioma inglés.

Palabras clave: Estrategia, Hands On, Incidencia, Desarrollo De La Comprensión Lectora, Inglés

\section{INTRODUCCIÒN}

Las estrategias metodológicas permiten identificar principios, criterios y procedimientos que configuran la forma de actuar del docente en relación con la programación, implementación y evaluación del proceso de enseñanza aprendizaje (Díaz, 2016).

De que proceso habla?Según Halten (1987), Es el proceso a través del cual una organización formula objetivos, y está dirigido a la obtención de los mismos. Estrategia es el medio, la vía, es el cómo para la obtención de los objetivos de la organización. Es el arte (maña) de entremezclar el análisis interno y la sabiduría utilizada por los dirigentes para crear valores de los recursos y habilidades que ellos controlan. Para diseñar una estrategia exitosa hay dos claves; hacer lo que hago bien y escoger los competidores que puedo derrotar. Análisis y acción están integrados en la dirección estratégica (p. 45). se mencionan la manera de usar la estrategia pero no se parafrasea, no hay un análisis de lo que el autor citado menciona, dentro del párrafo. Se hace mención de mucheas conceptos de estartegia y nunca el autor da su punto de vista sobre el concepto y la clase de ingles.

El autor Michael Porter (1995) con visión empresarial define a una estrategia competitiva en: "Desarrollar una amplia formula de cómo la 
empresa va a competir, cuáles deben ser sus objetivos y qué políticas serán necesarias para alcanzar tales objetivos."

Según Weinstein y Mayer (1986), "Las estrategias de aprendizaje pueden ser definidas como conductas y pensamientos que un aprendiz utiliza durante el aprendizaje con la intención de influir en su proceso de codificación" (p. 315).

Para Monereo (1994), las estrategias de aprendizaje son procesos de toma de decisiones (conscientes e intencionales) en los cuales el alumno elige y recupera, de manera coordinada, los conocimientos que necesita para cumplimentar una determinada demanda $\mathrm{u}$ objetivo, dependiendo de las características de la situación educativa en que se produce la acción.

Según Jaim Weitzman (2012) Es empelo de herramientas metodológicas capaces de gestar un genuino aprovechamiento de cada una de las instancias proclives al desarrollo autónomo del estudiante, tanto en la esfera personal como colectiva.

Según Melendres y Tocto (2010, pág. 14), la estrategia lúdica es una metodología de enseñanza de carácter participativo y dialógico impulsado por el uso creativo y pedagógicamente coherente, técnicas, ejercicios, historias y juegos educativos, creado específicamente para generar un aprendizaje significativo, tanto en términos de conocimientos, destrezas y habilidades sociales, como los valores de incorporación.

Y liego de improviso se cambia a 1 tema de las actividades lúdicas ... no hay coherencia $i$ o cohesion en a escritura $i$

Una actividad lúdica es realizada en el tiempo libre de los individuos, con el objetivo de liberar tensiones, huir de la rutina diaria y preocupaciones, para obtener un poco de placer, diversión y entretenimiento, así como otros beneficios, entre los cuales están (Kintsch, 1998):

- Amplía la expresión corporal.

- Desenvuelve la concentración y agilidad mental.

- Mejora el equilibrio y flexibilidad.

- Aumenta la circulación sanguínea.

- Libera endorfina y serotonina.

- Proporciona la inclusión social.

Las actividades lúdicas pueden ser variadas, como: ejercicios físicos, mentales, destreza, equilibrio, entre otros. En relación a los estudiantes, la práctica de un ejercicio lúdico es indispensable para su crecimiento personal y social, aparte de ser visto como una alternativa de ocio sana. Los beneficios que conlleva la realización de alguna actividad son: aumento de autoestima, desarrolla la creatividad y pensamiento, estimula la socialización, explora las posibilidades sensoriales y motoras, prepara al estudiante al mundo del 
trabajo, entre otras. (debo suponer que quien dice esto es el autor anterior o es un análisis de quein escrbe?)

El juego posee un objetivo educativo, se estructura como un juego reglado que incluye momentos de acción pre - reflexiva y de simbolización o apropiación abstracta - lógica de lo vivido para el logro de objetivos de enseñanza curriculares, cuyo objetivo último es la apropiación por parte del jugador, de los contenidos fomentando el desarrollo de la creatividad (Paula, 2008).

Y súbitamente se pasa a otro tema, no hay cohesión, son cosas sueltas... y ahora la comprensión lectora, uso de conectores y puntuación en los párrafos para unirlos y cambiar de tema al cambiar de párrafo.

La comprensión de lectura es una habilidad básica sobre la cual una serie de capacidades relacionadas se desarrollan: la gestión de la vía oral, amor por la lectura, así como un pensamiento crítico. El desarrollo de habilidades para la comprensión de la lectura es una forma de que la provisión de herramientas para la vida académica, profesional y social de los estudiantes (Fundacion BBVA, 2011).

La comprensión de lectura es un indicador clave a la hora de hacer planes de clases para el desarrollo de las autoridades gubernamentales; como un indicador sensible de una educación de calidad. Una persona que entiende lo que es la lectura que va a poder lograr un mejor desarrollo profesional, técnico y social.(el párrafo es incoherente, la lectura, los planes de clase y las autoridades, se percibe la idea pero la redacción es ambigua.)

Para desarrollar la comprensión lectora, en la guía se aplica una metodología didáctica modelo actual que se caracteriza por ser participativo y comunicativo. El docente mediante la guía orienta el aprendizaje de manera participativa, enseña, además de conocimiento habilidades y destrezas para ser autónomo en el aprendizaje.

El presente trabajo de investigación se basa en la Teoría del Juego como Anticipación Funcional. Según Karl Gross (1902). Hacer un ejercicio preparatorio (juego) será el inicio para adiestrar anticipadamente al educando para futuras capacidades serias. Este párrafo debería estar al inicio, y explicar cómo los juegos se adaptan a la lectura..

Para (Karl Gross) el juego es objeto de una investigación psicológica especial, siendo el primero en constatar el papel del juego como fenómeno de desarrollo del pensamiento y de la actividad. Para Gross el juego es uno de los elementos más importantes en el desarrollo, pues es un empuje para practicar los instintos, los cuales obligan al ser humano a ser activo y le impulsa a continuar desarrollándose. El juego simbólico surge en los niños/as a partir del pre ejercicio y les permitirá diferenciar la realidad de la ficción.

El autor menciona al juego con niños, el autor omite el análisis si el nivel de adultos en universidades puede traer el mismo resultado, no se 
relaciona comentarios a lo que la revisión de literatura trae. Si la RL es puramente lo que el universo sabe acerca del tema, pero la reacción debe llevar al lector hacia el entendimiento de lo que se busca con la investigación. El camino de lectura es escabroso y demasiado cambiante)

\section{METODOLOGÍA}

La investigación tiene un diseño transversal; los datos fueron recogidos en un solo momento. Es el estudio de un punto de corte específico en el tiempo, su propósito es describir las variables y analizar su impacto en un momento dado, y de la presente investigación se inició en 2014 y llevó a cabo su finalización en 2015.

La investigación es de tipo correlacional, dado que se determinó la incidencia de la estrategia hand on en relación a la incidencia de la comprensión de lectura del idioma inglés, además fue de campo ya que se desarrolló con los estudiantes del sexto nivel "E" del Centro de Idiomas de la UNACH. (porque hay una "relación de la incidencia"...it is overwording?)

Se aplicó el método hipotético deductivo, inductivo y analítico. Las partes y elementos de la investigación se analizaron mediante la observación de la muestra investigada para encontrar las causas, naturaleza y efectos del hecho y proponer la solución (guía didáctica "HANDS ON") para desarrollar la comprensión lectora del idioma inglés que favorecerá a docentes y estudiantes. ( Hasta qué punto está bien decir que se encontraron causa y efecto en la muestra observada, es eso lo que se busca?)

A través del método sintético, con todos los elementos obtenidos y analizados, se reconstruirá un todo, es decir hacer un resumen del trabajo investigativo para dar paso a las efectivas soluciones. ( Como se resume un trabajo investigativo con la reconstrucción de un todo?.. me parece aventura decir que se reconstruye un todo, y se usa un resumen? Para encontrar soluciones.. no es concreto)

La ficha de observación grupal fue mediante un test (neuropsi) para la recopilación de la información basados en indicadores y sub - indicadores. ( no se exlicca que es neuropsi)

Se estableció la población que integran los estudiantes del 6to. Semestre paralelo "E" del Centro de Idiomas de la Facultad de Ciencias de la Educación, Humanas y Tecnologías de la UNACH y debido a que la población utilizada en esta investigación es finita, limitada y manejable, se utilizó el total de la misma para la muestra. 
Tabla 1. Muestra

\begin{tabular}{|l|c|c|}
\hline CARACTERÍSTICAS & NÚMERO & PORCENTAJE \\
\hline Estudiantes & 30 & $100 \%$ \\
\hline TOTAL & 30 & $100 \%$ \\
\hline
\end{tabular}

Fuente: Secretaría - UNACH.

Elaborado por: Pazmiño Luis, 2015

Ami forma de ver no hace falta exponer la muestra en un cuadro, porque?

El procedimiento para el análisis de los resultados se lleva a cabo de la siguiente manera:

- Validación del instrumento o de pilotaje. La aplicación de la encuesta o el tipo de instrumento que se haga.

- Recolección de la información.

- Tabulación de la información pregunta por pregunta.

- Procesamiento de gráficos utilizando el programa Microsoft Excel 2010.

- Análisis e interpretación de los datos.

- Sacar conclusiones y la redacción de recomendaciones.

- La prueba de hipótesis que se hace de una forma cualitativa y cuantitativamente.

De qué forma se usan los métodos cuantitativos y cualitativos para probar la hipótesis?.. se debe mencionar)

Para alcanzar la meta en la presente investigación se elaboró e implementó la guía didáctica " HANDS ON", cuyos contenidos están enfocados en el uso de cuentos, juegos lúdicos y vocabulario a fin de influir en el desarrollo de la comprensión lectora del idioma inglés en los estudiantes de sexto nivel " E " del Centro de Idiomas de la UNACH.

Porque se usan comillas, y mayúsculas, que es lo correcto?

\section{RESULTADOS Y DISCUSIÓN}

El proceso metodológico para instituir la ficha de observación en la cual se pretende analizar e interpretar los resultados antes y después de aplicar la guía didáctica "Hands ON" se realizó un Test estandarizado de comprensión lectora, pues como entendemos la comprensión lectora es un fenómeno inobservable, y es un fenómeno interactivo: depende de la competencia y los conocimientos del lector, pero también de las características del texto que está leyendo. Por lo tanto, ninguna medida nos va a decir cómo es la comprensión de un lector, sino que nos van a proporcionar una información indirecta a partir de la cual se deberá hacer un juicio sobre cómo es esa comprensión. Dicho esto, se aplica a los estudiantes del 6to. Semestre 
del centro de Idiomas un test estandarizado y moderno para la evaluación de la comprensión lectora en inglés.

Porque se dice que el fenómeno es inobservable?

El test aplicado (anexos) se adapta al conocido Neuropsi - que apareció a finales del 2013 se caracteriza por evaluar la orientación, atención y concentración, lenguaje, memoria, funciones ejecutivas, lectura, escritura y calculo. Una vez evaluados los estudiantes se analiza las características individuales de cada uno y se hace un análisis a interpretación de datos.

Tabla 2. Resultados finales antes de aplicar la guía

RESULTADOS FINALES DEL DESARROLLO DE LA COMPRENSIÓN LECTORA ANTES DE APLICAR LA GUÍA DIDÁCTICA“HANDS ON"

\begin{tabular}{|c|c|c|c|c|c|c|c|c|c|c|c|c|c|c|}
\hline \multirow{3}{*}{ SUBINDICADOR } & \multicolumn{14}{|c|}{ INDICADORES } \\
\hline & \multicolumn{6}{|c|}{ JUEGOS } & \multicolumn{5}{|c|}{ HISTORIAS } & \multicolumn{3}{|c|}{ VOCABULARIO } \\
\hline & P1 & $\mathrm{P} 2$ & P3 & $\mathrm{P} 4$ & P5 & P6 & P7 & P8 & P9 & P10 & P11 & $\mathrm{P} 12$ & $\mathrm{P} 13$ & P14 \\
\hline SI & 5 & 4 & 5 & 6 & 4 & 7 & 5 & 6 & 4 & 5 & 4 & 5 & 8 & 6 \\
\hline A VECES & 7 & 6 & 8 & 5 & 6 & 5 & 5 & 10 & 8 & 9 & 8 & 8 & 9 & 9 \\
\hline $\mathrm{NO}$ & 18 & 20 & 17 & 19 & 20 & 18 & 20 & 14 & 18 & 16 & 18 & 17 & 13 & 15 \\
\hline TOTAL & 30 & 30 & 30 & 30 & 30 & 30 & 30 & 30 & 30 & 30 & 30 & 30 & 30 & 30 \\
\hline
\end{tabular}

Fuente: Ficha de observación estudiantes 6to. nivel, Centro de Idiomas- UNACH

Elaborado por: Autores

Tabla 3. Resultados finales después de aplicar la guía

\begin{tabular}{|c|c|c|c|c|c|c|c|c|c|c|c|c|c|c|}
\hline \multicolumn{15}{|c|}{$\begin{array}{l}\text { RESULTADOS FINALES DEL DESARROLLO DE LA COMPRENSIÓN LECTORA } \\
\text { DESPUÉS DE APLICAR LA GUÍA DIDÁCTICA "HANDS ON" }\end{array}$} \\
\hline \multirow{3}{*}{ SUBINDICADOR } & \multicolumn{14}{|c|}{$\begin{array}{l}\text { INDICADORES } \\
\end{array}$} \\
\hline & \multicolumn{6}{|c|}{ JUEGOS } & \multicolumn{5}{|c|}{ HISTORIAS } & \multicolumn{3}{|c|}{ VOCABULARIO } \\
\hline & P1 & P2 & P3 & P4 & P5 & P6 & P7 & P8 & P9 & $\mathrm{P} 10$ & P11 & $\mathrm{P} 12$ & $\mathrm{P} 13$ & $\mathrm{P} 14$ \\
\hline SI & 19 & 21 & 18 & 17 & 19 & 16 & 17 & 20 & 18 & 17 & 19 & 16 & 20 & 17 \\
\hline A VECES & 6 & 5 & 7 & 8 & 7 & 8 & 7 & 7 & 7 & 7 & 7 & 6 & 6 & 7 \\
\hline NO & 5 & 4 & 5 & 5 & 4 & 6 & 6 & 3 & 5 & 6 & 7 & 8 & 4 & 6 \\
\hline TOTAL & 30 & 30 & 30 & 30 & 30 & 30 & 30 & 30 & 30 & 30 & 33 & 30 & 30 & 30 \\
\hline
\end{tabular}

Fuente: Ficha de observación estudiantes 6to. nivel, Centro de Idiomas- UNACH

Elaborado por: Pazmiño Luis, 2015

Tabla 4. Resultado final porcentual antes y después de aplicar la guía

\begin{tabular}{ccccccccc}
\hline & \multicolumn{4}{c}{ ANTES } & \multicolumn{4}{c}{ DESPUÉ } \\
INDICADOR & SI & $\begin{array}{c}\text { A } \\
\text { VECES }\end{array}$ & NO & TOTAL & SI & A & NO & TOTAL \\
\hline P1 & $17 \%$ & $23 \%$ & $60 \%$ & $100 \%$ & $63 \%$ & $20 \%$ & $17 \%$ & $100 \%$ \\
P2 & $13 \%$ & $20 \%$ & $67 \%$ & $100 \%$ & $70 \%$ & $17 \%$ & $13 \%$ & $100 \%$ \\
P3 & $17 \%$ & $27 \%$ & $56 \%$ & $100 \%$ & $60 \%$ & $23 \%$ & $17 \%$ & $100 \%$ \\
P4 & $20 \%$ & $17 \%$ & $63 \%$ & $100 \%$ & $56 \%$ & $27 \%$ & $17 \%$ & $100 \%$ \\
P5 & $13 \%$ & $20 \%$ & $67 \%$ & $100 \%$ & $63 \%$ & $23 \%$ & $14 \%$ & $100 \%$ \\
P6 & $23 \%$ & $17 \%$ & $60 \%$ & $100 \%$ & $53 \%$ & $27 \%$ & $20 \%$ & $100 \%$ \\
P7 & $17 \%$ & $17 \%$ & $66 \%$ & $100 \%$ & $57 \%$ & $23 \%$ & $20 \%$ & $100 \%$ \\
P8 & $20 \%$ & $33 \%$ & $47 \%$ & $100 \%$ & $67 \%$ & $23 \%$ & $10 \%$ & $100 \%$ \\
\hline
\end{tabular}




\begin{tabular}{ccccccccc}
\hline P9 & $13 \%$ & $27 \%$ & $60 \%$ & $100 \%$ & $60 \%$ & $23 \%$ & $17 \%$ & $100 \%$ \\
P10 & $17 \%$ & $30 \%$ & $53 \%$ & $100 \%$ & $57 \%$ & $23 \%$ & $20 \%$ & $100 \%$ \\
P11 & $13 \%$ & $27 \%$ & $60 \%$ & $100 \%$ & $53 \%$ & $23 \%$ & $24 \%$ & $100 \%$ \\
P12 & $17 \%$ & $27 \%$ & $56 \%$ & $100 \%$ & $53 \%$ & $20 \%$ & $27 \%$ & $100 \%$ \\
P13 & $27 \%$ & $30 \%$ & $43 \%$ & $100 \%$ & $67 \%$ & $20 \%$ & $13 \%$ & $100 \%$ \\
P14 & $20 \%$ & $30 \%$ & $50 \%$ & $100 \%$ & $57 \%$ & $23 \%$ & $20 \%$ & $100 \%$ \\
\hline
\end{tabular}

Fuente: Ficha de observación estudiantes 6to. nivel, Centro de Idiomas- UNACH

Elaborado por: Pazmiño Luis, 2015

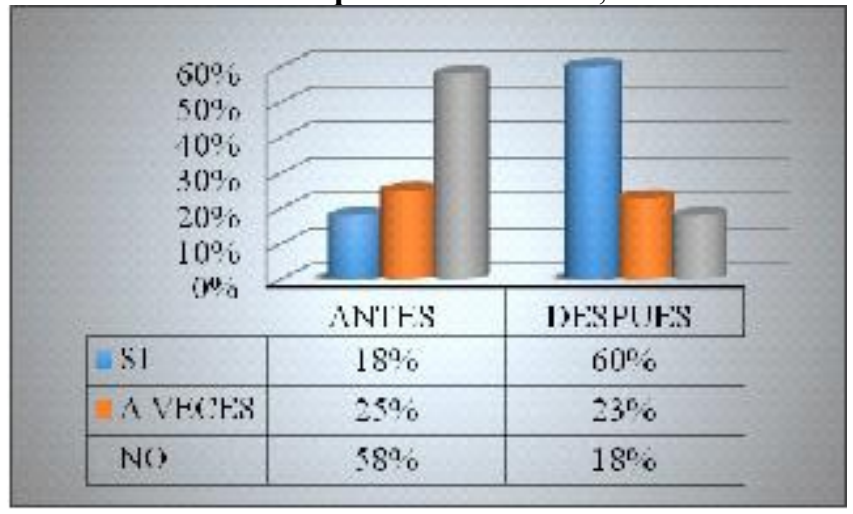

Gráfico 1. Resultados finales antes y después de la aplicación de la guía.

\section{Comprobación de la hipótesis específica 1}

- $\quad \pi 1$ : Corresponde al porcentaje del SI del resultado del desarrollo de la comprensión lectora utilizando los cuentos, después de la aplicación de la guía.

- $\pi 2$ : Corresponde al porcentaje del SI del resultado del desarrollo de la comprensión lectora utilizando los cuentos, antes de la aplicación de la guía de $\pi 2$ :

Pruebe que el porcentaje de $\pi 1$ es significativamente superior al porcentaje

\section{Planteamiento de la hipótesis}

La aplicación de una guía didáctica " HANDS ON", con el uso de cuentos influye en el desarrollo de la comprensión lectora del idioma inglés en los estudiantes de sexto nivel "E" del Centro de Idiomas de la UNACH, período 2014-2015.

\begin{tabular}{ccc}
\hline SUBINDICADOR & ANTES & DESPUÉS \\
\hline SI & $17 \%$ & $61 \%$ \\
A VECES & $21 \%$ & $23 \%$ \\
NO & $62 \%$ & $16 \%$ \\
TOTAL & $100 \%$ & $100 \%$ \\
\hline
\end{tabular}

Fuente: Aplicaciòn de la guía - Hipótesis 1

Elaborado por: Pazmiño Luis, 2015 
- Ho: $\pi 1=\pi 2$ (No hay diferencia entre los porcentajes del SI de $\pi 1$ y de $\pi 2)$

- Ha: $\pi 1>\pi 2$ (El porcentaje del SI de $\pi 1$ es significativamente superior que el porcentaje de $\pi 2)$.

\section{Nivel de significación}

$\alpha=0.05$ es la probabilidad de cometer el error tipo I (rechazar la hipótesis alternativa siendo esta verdadera, como el valor es pequeño 0.05 es muy difícil que ocurra este error).

\section{Criterio}

Rechace la Ho si Zc $>1.645$ valor de prueba en la curva de cola superior.

\begin{tabular}{|c|c|c|}
\hline NIVEL DE SIGNIFICACIÓN & 0.05 & 0.01 \\
\hline Valores críticos de Z para ensayos de una cola & \pm 1.645 & \pm 2.33 \\
\hline Valores críticos de Z para ensayos de dos colas & \pm 1.96 & \pm 2.58 \\
\hline
\end{tabular}

Zc es el valor calculado de z que se obtiene aplicando la fórmula:

$$
Z_{c}=\frac{\bar{X}_{1}-\bar{X}_{2}}{\sqrt{\frac{\bar{X}_{1} q_{1}}{n 1}+\frac{\bar{X}_{2} q_{1}}{n 2}}}
$$

\section{Cálculos}

- $\bar{X}_{1}=0.61$ (es la media del porcentaje del SI, después de la aplicación de la guía "Hands On" con el uso de cuentos para el desarrollo de la comprensión lectora.)

- $\bar{X}_{2}=0.17$ (es la media del porcentaje del SI, antes de la aplicación de la guía "Hands On" con el uso de cuentos para el desarrollo de la comprensión lectora.)

$$
\begin{gathered}
\mathrm{q} 1=1-0.61=0.39 \\
\mathrm{q} 2=1-0.17=0.83 \\
\mathrm{n} 1=\mathrm{n} 2=30 \\
Z_{c}=\frac{0.61-0.17}{\sqrt{\frac{(0.61)(0.39)}{30}+\frac{(0.17)(0.83)}{30}}}=\frac{0.44}{\sqrt{\frac{0.237}{30}+\frac{0.141}{30}}}=\frac{0.44}{\sqrt{0,0126}}= \\
Z_{c}=\frac{0.44}{\sqrt{0,0126}}=\frac{0.44}{01122}=3.921
\end{gathered}
$$




\section{Decisión}

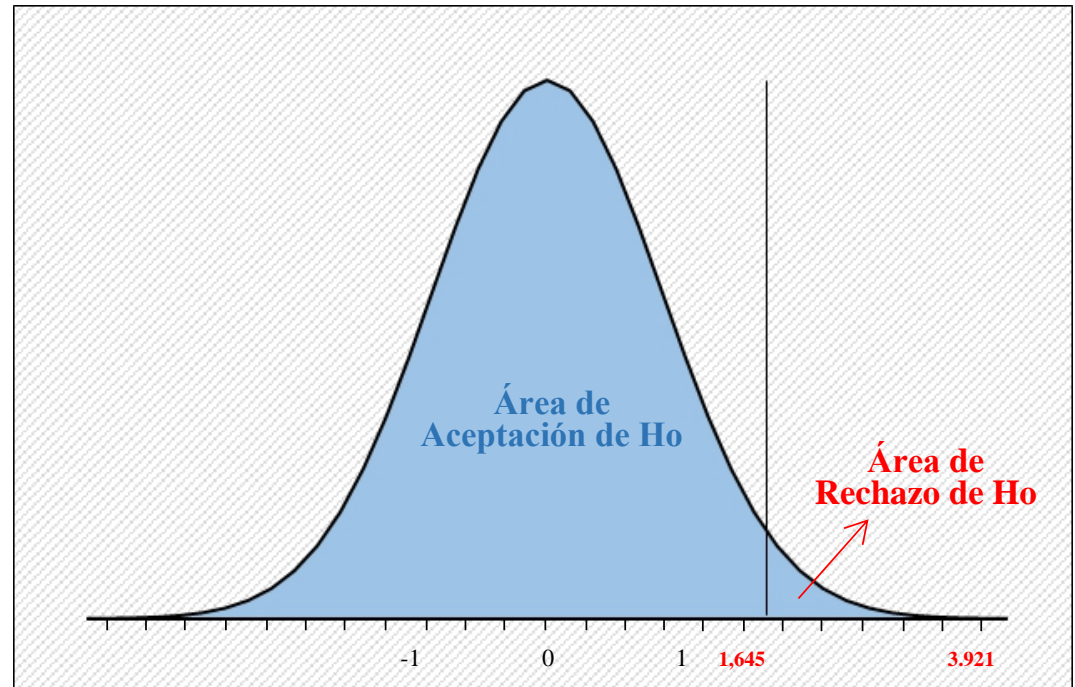

$$
\mathrm{Zc}=3.921>1.645=\mathrm{zt}
$$

Como Zc es mayor que Z, si cae en el área de rechazo, por tanto, si hay suficiente evidencia para rechazar Ho (Hipótesis Nula) y aceptar Ha (Hipótesis alternativa).

\section{Comprobación de la hipótesis específica 2}

- $\pi 1$ : Corresponde al porcentaje del SI del resultado del desarrollo de la comprensión lectora utilizando los juegos, después de la aplicación de la guía.

- $\pi 2$ : Corresponde al porcentaje del SI del resultado del desarrollo de la comprensión lectora utilizando los cuentos, antes de la aplicación de la guía.

Pruebe que el porcentaje de $\pi 1$ es significativamente superior al porcentaje de $\pi 2$ :

\section{Planteamiento de la hipótesis}

La aplicación de una guía didáctica " HANDS ON", con el uso de juegos influye en el desarrollo de la comprensión lectora del idioma inglés en los estudiantes de sexto nivel "E" del Centro de Idiomas de la UNACH, Riobamba, período 2014-2015.

\begin{tabular}{ccc}
\hline SUBINDICADOR & ANTES & DESPUÉS \\
\hline SI & $16 \%$ & $59 \%$ \\
A VECES & $27 \%$ & $23 \%$ \\
NO & $57 \%$ & $18 \%$ \\
TOTAL & $100 \%$ & $100 \%$ \\
\hline
\end{tabular}


- Ho: $\pi 1=\pi 2$ (No hay diferencia entre los porcentajes del SI de $\pi 1$ y de $\pi 2)$

- Ha: $\pi 1>\pi 2$ (El porcentaje del SI de $\pi 1$ es significativamente superior que el porcentaje de $\pi 2$ ).

\section{Nivel de significación}

$\alpha=0.05$ es la probabilidad de cometer el error tipo I (rechazar la hipótesis alternativa siendo esta verdadera, como el valor es pequeño 0.05 es muy difícil que ocurra este error).

\section{Criterio}

Rechace la Ho si Zc $>1.645$ valor de prueba en la curva de cola superior.

\begin{tabular}{|c|c|c|}
\hline NIVEL DE SIGNIFICACIÓN & 0.05 & 0.01 \\
\hline Valores críticos de Z para ensayos de una cola & \pm 1.645 & \pm 2.33 \\
\hline Valores críticos de Z para ensayos de dos colas & \pm 1.96 & \pm 2.58 \\
\hline
\end{tabular}

Zc es el valor calculado de z que se obtiene aplicando la fórmula:

$$
Z_{c}=\frac{\bar{X}_{1}-\bar{X}_{2}}{\sqrt{\frac{\bar{X}_{1} q_{1}}{n 1}+\frac{\bar{X}_{2} q_{1}}{n 2}}}
$$

\section{Cálculos}

- $\bar{X}_{1}=0.59$ (es la media del porcentaje del SI, después de la aplicación de la guía "Hands On" con el uso de juegos para el desarrollo de la comprensión lectora.)

- $\bar{X}_{2}=0.16 \quad$ (es la media del porcentaje del SI, antes de la aplicación de la guía "Hands On" con el uso de juegos para el desarrollo de la comprensión lectora.)

$$
\begin{aligned}
& \mathrm{q} 1=1-0.59=0.41 \\
& \mathrm{q} 2=1-0.16=0.84 \\
& \mathrm{n} 1=\mathrm{n} 2=30 \\
& Z_{c}=\frac{0.59-0.16}{\sqrt{\frac{(0.59)(0.41)}{30}+\frac{(0.16)(0.84)}{30}}}=\frac{0.44}{\sqrt{\frac{0.241}{30}+\frac{0.134}{30}}}=\frac{0.43}{\sqrt{0,0124}}= \\
& Z_{c}=\frac{0.43}{01113}=3.805
\end{aligned}
$$




\section{Decisión}

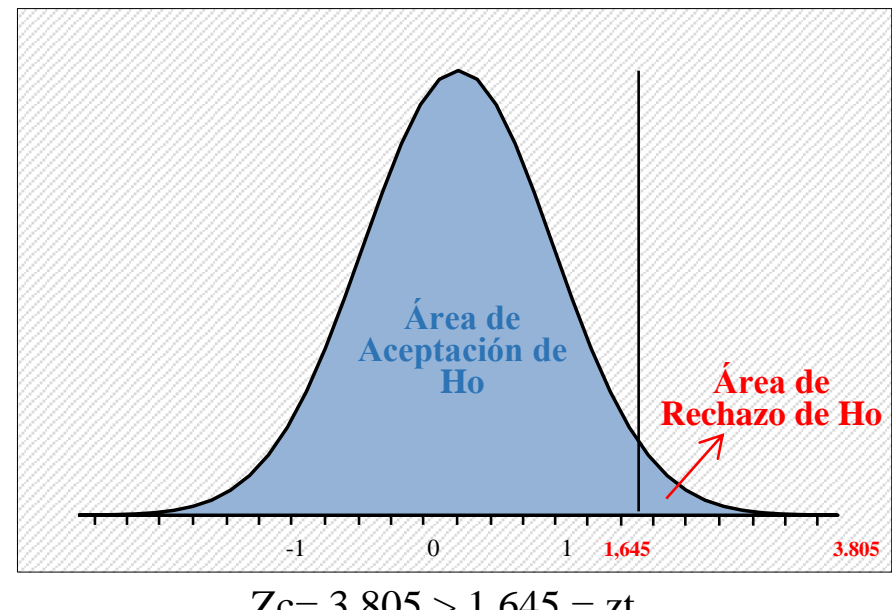

Como Zc es mayor que Z, si cae en el área de rechazo, por tanto, si hay suficiente evidencia para rechazar Ho (Hipótesis Nula) y aceptar Ha (Hipótesis alternativa).

\section{Comprobación de la hipótesis específica 3}

- $\pi 1$ : Corresponde al porcentaje del SI del resultado del desarrollo de la comprensión lectora utilizando el vocabulario, después de la aplicación de la guía.

- $\pi 2$ : Corresponde al porcentaje del SI del resultado del desarrollo de la comprensión lectora utilizando vocabulario, antes de la aplicación de la guía.

Pruebe que el porcentaje de $\pi 1$ es significativamente superior al porcentaje de $\pi 2$ :

\section{Planteamiento de la hipótesis}

La aplicación de una guía didáctica " HANDS ON", con el uso de vocabulario influye en el desarrollo de la comprensión lectora del idioma inglés en los estudiantes de sexto nivel "E" del Centro de Idiomas de la UNACH, Riobamba, período 2014-2015.

\begin{tabular}{ccc}
\hline SUBINDICADOR & ANTES & DESPUÉS \\
\hline SI & $21 \%$ & $59 \%$ \\
A VECES & $29 \%$ & $21 \%$ \\
NO & $50 \%$ & $20 \%$ \\
TOTAL & $100 \%$ & $100 \%$ \\
\hline
\end{tabular}

- Ho: $\pi 1=\pi 2$ (No hay diferencia entre los porcentajes del SI de $\pi 1$ y de $\pi 2)$

- Ha: $\pi 1>\pi 2$ (El porcentaje del SI de $\pi 1$ es significativamente superior 
que el porcentaje de $\pi 2$ ).

\section{Nivel de significación}

$\alpha=0.05$ es la probabilidad de cometer el error tipo I (rechazar la hipótesis alternativa siendo esta verdadera, como el valor es pequeño 0.05 es muy difícil que ocurra este error).

\section{Criterio}

Rechace la Ho si Zc > 1.645 valor de prueba en la curva de cola superior.

Valores críticos de $\mathrm{Z}$ para ensayos de una cola

Valores críticos de $\mathrm{Z}$ para ensayos de dos colas

\begin{tabular}{|l|c|c|} 
NIVEL DE SIGNIFICACIÓN & 0.05 & 0.01 \\
\hline
\end{tabular}

$+1.645$

$+1.96$

$\pm 2.58$

Zc es el valor calculado de $\mathrm{z}$ que se obtiene aplicando la fórmula:

$$
Z_{c}=\frac{\bar{X}_{1}-\bar{X}_{2}}{\sqrt{\frac{\bar{X}_{1} q_{1}}{n 1}+\frac{\bar{X}_{2} q_{1}}{n 2}}}
$$

\section{Cálculos}

- $\bar{X}_{1}=0.59$ (es la media del porcentaje del SI, después de la aplicación de la guía "Hands On" con el uso de vocabulario para el desarrollo de la comprensión lectora.)

- $\quad \bar{X}_{2}=0.20$ (es la media del porcentaje del SI, antes de la aplicación de la guía "Hands On" con el uso de vocabulario para el desarrollo de la comprensión lectora.)

$$
\begin{gathered}
\mathrm{q} 1=1-0.59=0.41 \\
\mathrm{q} 2=1-0.20=0.80 \\
\mathrm{n} 1=\mathrm{n} 2=30 \\
Z_{c}=\frac{0.59-0.20}{\sqrt{\frac{(0.59)(0.41)}{30}+\frac{(0.20)(0.80)}{30}}}=\frac{0.39}{\sqrt{\frac{0.241}{30}+\frac{0.160}{30}}}=\frac{0.39}{\sqrt{0,0133}}= \\
Z_{c}=\frac{0.39}{01153}=3.382
\end{gathered}
$$




\section{Decisión}

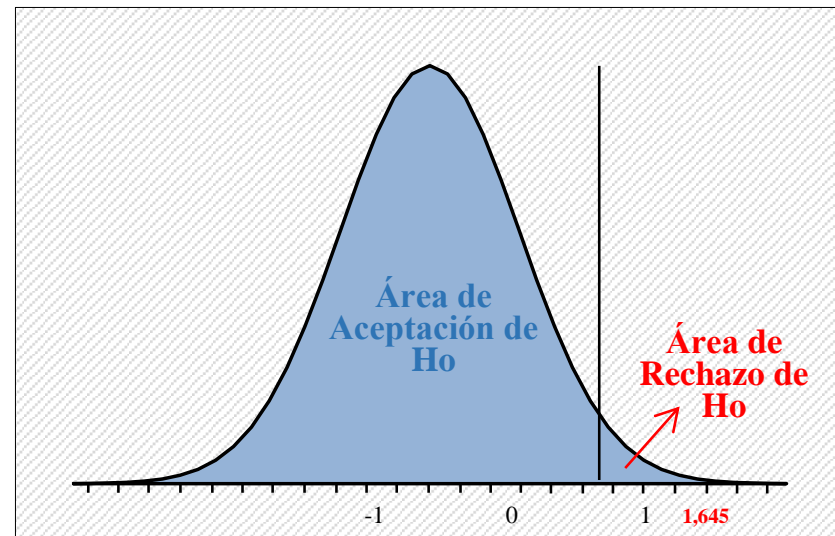

Como Zc es mayor que Z, si cae en el área de rechazo, por tanto, si hay suficiente evidencia para rechazar Ho (Hipótesis Nula) y aceptar Ha (Hipótesis alternativa).

\section{CONCLUSION}

- Una vez que se realizó la investigación, se obtuvo conclusiones preliminares: deficiencia en la comprensión lectora del idioma inglés. Al aplicar la guía didáctica "Hands ON" (cuentos, juegos y vocabulario) propuesta en este trabajo se concluye que efectivamente, favorece a desarrollar la comprensión lectora del idioma inglés en los estudiantes del 6to nivel "E" del Centro de Idiomas de la UNACH.

- La metodología utilizada en la guía responde a la necesidad del estudiante y profesor de obtener un aprendizaje significativo y el de brindar efectivamente los conocimientos que posee el docente respecto a los niveles de la comprensión lectora del idioma inglés, literal, inferencial, reconstructivo y apreciativo.

- Las historias, juegos interactivos y vocabulario propuesto en la guía despiertan el interés del estudiante, lo motivan a seguir aprendiendo el idioma inglés, han asistido con regularidad a las clases por lo tanto han mejorado su capacidad de síntesis y el aumento del léxico.

- Alrededor del 70\% de estudiantes después de aplicar la guía didáctica presentaron actitudes y aptitudes positivas para comprender textos en inglés evidenciándose un mayor nivel de capacidad crítica en sus lecturas.

- La aplicación de esta guía sirve como ayuda en el trabajo del docente, más no reemplaza a las asignaturas dictadas en el aula.

\section{RECOMENDACIONES}

- Para entender el porqué de resultados negativos en la enseñanza del idioma 
inglés se recomienda indagar en primer lugar, las capacidades intelectuales de los estudiantes en segundo lugar las metodologías utilizadas por el docente.

- Cuando un docente facilita material educativo, es recomendable que este contenga metodologías lúdicas y más si se trata del idioma inglés, pues como sabemos es difícil adquirir el nuevo idioma cuando el estudiante es una persona adulta, no son todos los casos, pero si en su gran mayoría.

- Es recomendable que el docente aplique la guía desde el inicio de clases con ello, los estudiantes responderán de manera significativa a los contenidos de la asignatura. Porque habrá antecedentes para comprender los textos en inglés, tienen despierta su capacidad crítica.

- Para el docente, se recomienda tener más énfasis o más interés en aquellos estudiantes con muy baja capacidad cognitiva y tener en cuenta a la hora de elaborar los materiales didácticos, lograr en un estudiante a tener seguridad en su capacidad intelectual es un reto para todo docente.

- Se recomienda utilizar la guía didáctica como apoyo en el desarrollo de la asignatura, es necesario y muy recomendable ampliar las actividades de cada unidad con el fin de lograr aprendizajes significativos.

\section{References:}

1. Díaz, A. (2016, Marzo 16). Galeon.com hispavista. Retrieved from Estratégias Metodológicas: http://aureadiazgonzales.galeon.com/index.html.

2. Fundacion BBVA. (2011). ¿Por qué es importante la comprensión lectora? Retrieved from http://www.leer.pe/ique-hacemos/ipor-quees-importante-la-comprension-lectora.

3. Halten, K. J. (1987).

4. Monereo, C. (1994). Estrategias de enseñanza y aprendizaje. Formación del profesorado y aplicación en la escuela. Barcelona: Graó.

5. Porter, M. (1995). Ventajas competitivas . New York: Free Press.

6. Weinstein, C. Y. (1986). The teaching of learning strategies Handbook of research on teaching. New York : McMillan.

7. Weitzman, J. (2012, Septiembre 28). Educrea . Retrieved from Educrea : http://educrea.cl/medios-audiovisuales-2/ 